\title{
The benefit of statins in SARS-CoV-2 patients: further metabolic and prospective clinical studies are needed
}

\author{
Maurizio Bifulco ${ }^{1}{ }^{1}$ - Michele Ciccarelli ${ }^{2}$ - Dario Bruzzese ${ }^{3}$ - Andrea Dipasquale ${ }^{2,4}$ - Andrea G. Lania ${ }^{4,5}$. \\ Gherardo Mazziotti $^{4,5} \cdot$ Patrizia Gazzerro ${ }^{6}$
}

Received: 15 September 2020 / Accepted: 5 November 2020 / Published online: 20 November 2020

(c) Springer Science+Business Media, LLC, part of Springer Nature 2020

The severe acute respiratory syndrome coronavirus 2 (SARS-CoV-2) is a novel enveloped RNA beta-coronavirus responsible for the coronavirus disease-19 (COVID-19) ranging from asymptomatic cases to severe respiratory involvement [1]. The first clinical case of COVID-19 in Italy was diagnosed on February 20th 2020, then the infection has rapidly spread and Lombardy became the epicenter of the COVID-19 pandemic in Italy.

The potential of cholesterol-lowering drug statin use to counteract SARS-CoV-2 mortality and the mechanisms triggered by these compounds in infections, has been suggested by our group [2,3]. A recent retrospective study by Zhang et al. [4] performed in a very large cohort of 13,981 patients hospitalized for COVID-19 in Hubei Province, China, highlighted a reduced mortality risk in statin user patients applying impressive multiple statistical models, used to adjust for potential bias, and sensitivity analyses to limit the overall unmeasured confounders. The results

Maurizio Bifulco

maubiful@unina.it

$\triangle$ Patrizia Gazzerro

pgazzerro@unisa.it

1 Department of Molecular Medicine and Medical Biotechnologies, University of Naples "Federico II", 80131 Naples, Italy

2 Pneumology Unit, Humanitas Clinical and Research Center IRCCS, 20089 Rozzano, Milan, Italy

3 Department of Public Health, University of Naples Federico II, Naples, Italy

4 Endocrinology, Diabetology and Medical Andrology Unit, Humanitas Clinical and Research Center, IRCCS, 20089 Rozzano, Milan, Italy

5 Department of Biomedical Sciences, Humanitas University, 20090 Pieve Emanuele, Milan, Italy

6 Department of Pharmacy, University of Salerno, 84084 Fisciano (SA), Italy obtained by Zhang and coworkers highlighted that inhospital use of statins, compared to non-statin use, is significantly associated with a lower risk for all-cause death possible ascribable to the decreased inflammatory response during the hospitalization period. These data represent a strong and convincingly evidence supporting the working hypothesis of pleiotropic effects of statins in COVID-19. We are now retrospectively analyzing a cohort of 541 patients with COVID-19 (37\% females; overall mean age 65 years) admitted to Humanitas Clinical and Research Hospital (Rozzano, Milan, Italy) from March first 2020 to May 31st 2020. 75\% of patients required oxygenation support, $24.4 \%$ non invasive mechanical ventilation with CPAP (Continuous Positive Airway Pressure), 12.4\% invasive mechanical ventilation through orotracheal intubation (OTI). To identify factors associated with COVID19-related mortality, we analyzed several clinical and biochemical parameters investigating their correlation with a potential protective role of statins used by the patients before and after hospitalization for COVID-19. We also retrospectively analyzed a cohort of 86 patients $(46.5 \%$ female; overall mean age 75 years) admitted to Humanitas Clinical and Research Hospital from January 2019 to October 2019 for severe pneumonia or ARDS induced by causes other than SARS-CoV-2 infection.

The first end-point of the study was the evaluation of impact of treatment with statins on in-hospital COVID-19related mortality. As secondary end-points we also explored the association between serum cholesterol values and SARS-CoV-2 infection and outcome of COVID-19.

The retrospective study was approved by the Ethical Committee of Humanitas Clinical and Research Center, IRCCS, and the patients gave their consent to use the clinical and biochemical data for research purposes. In our cohort of COVID-19 patients, 117 (21.6\%) subjects were on statins therapy at admission. Statin users were older (72.9 \pm 10.9 years vs $63 \pm 14.5$ years; $p<0.001)$, more frequently smokers $(18.8$ vs $6.4 \% ; p<0.001)$, with a higher prevalence 
of diabetes (46.2 vs. $17.9 \% ; p<0.001)$, hypertension $(76.9$ vs. $43.3 \% ; p<0.001)$, chronic obstructive pulmonary disease (29.9 vs. $7.9 \% ; p<0.001)$, dyslipidemia (78.6 vs. $5.5 \% ; p<0.001)$ and cardiovascular disease (59 vs. $10.5 \%$; $p<0.001)$, higher procalcitonin values at admission $(0.26 \mathrm{ng} / \mathrm{mL}$; from 0.05 to 57 vs. $0.16 \mathrm{ng} / \mathrm{mL}$; from 0.05 to $32 ; p=0.003)$ and, as expected, lower level of total cholesterol $(150.1 \pm 48.4 \mathrm{mg} / \mathrm{dl}$ vs. $170.4 \pm 46.7 \mathrm{mg} / \mathrm{dl} ; p=$ $0.026)$ and LDL-cholesterol $(84 \pm 39.8 \mathrm{mg} / \mathrm{dl}$ vs. $104.9 \pm$ $37.6 \mathrm{mg} / \mathrm{dl} ; p=0.006)$.

In the overall cohort, a total of $123(22.7 \%)$ deaths were observed; the odds of all-cause mortality, when adjusting the analysis for age, gender, smoking habit, pre-existing comorbidities (e.g. dyslipidemia, diabetes, hypertension, cardiovascular disease), indicators of disease severity and organ injuries and blood biomarkers, were lower, although not significantly, in statin users with respect to non-statin users (adjusted odds ratio [aOR]: $0.75 ; 95 \%$ confidence interval [C.I.]: $0.26-2.17 ; p=0.593)$. The only factors that preserved a significant association with death were: age at admission (aOR: 1.15; 95\% C.I.: $1.11-1.2 ; p<0.001$ ), LDH (Lactate Dehydrogenase, aOR: 2.94; 95\% C.I.: 1.1-7.85; $p=0.031$ ) and ferritin levels (aOR: 1.45; 95\% C.I.: 1.03-2.05; $p=0.034)$.

When excluding from the analysis patients who required orotracheal intubation in whom statin treatment was discontinued, the odds of all-cause mortality in statin users were $14 \%$ lower than non-statin users (aOR: 0.86; $95 \%$ C.I.: $0.28-2.63 ; p=0.795)$, although the difference was still not significant.

Despite we failed to demonstrate a significant association between statin-use and reduced mortality risk, we cannot exclude that statin treatment may have a protective role in preventing serious clinical outcome of COVID-19 and weakening clinical symptoms of SARS-CoV-2 infection, such as already suggested [4, 5]. Unlike Zhang's study where statin treatment started at the day of hospital admission [4], our patients were usual users of statins which were prescribed for conditions other than SARS-CoV-2 and they were not withdrawn in most of the cases during the observation period. However, the lack of a significant association between statin-use and the favorable outcome of COVID 19 (e.g., reduced mortality risk) could be ascribable to the advanced clinical profiles (ranging from moderate/severe to critical) in our patients and the lack of information about the statin use in patients with SARS-CoV-2 infection who were not hospitalized and enrolled in the study. Moreover, it is reasonable to hypothesize that, among other, type of statin, timing, doses and indications for therapeutic prescription could be a not negligible source of bias in our patients.

In SARS-CoV-2 patients, lipid profile was reported to be altered in close relationship with the outcome of COVID-19 [6]. Specifically, a significant decrease of HDL-cholesterol was found in critical patients as compared with mild and severe cases. In our cohort, we did not found substantially differences in HDL-cholesterol levels between survivors and not survivors $(36.7 \pm 10.1$ and $37.2 \pm 11.8 \mathrm{mg} / \mathrm{dL}$, respectively; $p=0.867$ ) but, not surprisingly, the mean HDL-cholesterol values in both discharged and deceased subjects seem similar to those obtained in subjects with critical clinical profiles by Wei et al. [6]. These data strongly support more homogeneous and advanced clinical features in the patients we observed.

In a small group of patients $(n=59)$ from our SARSCoV-2 cohort in whom longitudinal evaluations of lipid profile was available, the HDL-cholesterol levels at the time of hospitalization resulted significantly lower than that obtained, in the 6-12 months before SARS-CoV2 infection (mean difference: $-6.4 \mathrm{mg} / \mathrm{dl}$; $95 \%$ C.I.: -11.7 to -1.1 ; $p=0.02$ ), without significant changes in total cholesterol and triglycerides values (data not shown).

Interestingly, after adjusting for sex and age, serum HDL-cholesterol levels resulted to be significantly lower in patients hospitalized for COVID-19 as compared to the control group of patients hospitalized in 2019 for pneumonia or ARDS induced by causes other than SARS-CoV-2 infection, $(36.9 \pm 10.4 \mathrm{mg} / \mathrm{dL}$ vs, $41.6 \pm 14.9 \mathrm{mg} / \mathrm{dl} ; p=$ $0.013)$ again without significant differences in total cholesterol $(165.2 \pm 47.8$ vs. $166.1 \pm 49, p=0.395)$ and triglycerides $(137.5 \pm 62.4$ vs. $119.1 \pm 57.6 ; p=0.058)$. These findings are consistent with the evidence from literature suggesting a significant role of lipid dysmetabolism as a predisposing factor, even if not critical, for severe outcomes in SARS-CoV-2 infection. Indeed, a recent study highlighted that SARS-CoV-2 triggers a metabolic reprogramming characterized by dysregulation of multiple apolipoproteins (such as APOA1 and APOM), reduction of serum bilirubin and lipid levels, increase of acute phase proteins and VCAM-1 [7]. In this context, HDLs lipoproteins behave as anti-inflammatories and antivirals through the direct neutralization of viruses and the interaction with ABCG1, a lipid transporter (expressed in alveolar macrophages, pneumocytes, $\mathrm{T}$ lymphocytes and dendritic cells) responsible for lung lipid homeostasis and acting as protective factor during infections [8, 9]. From this point of view, HDL-cholesterol could be an interesting parameter to evaluate in SARS-CoV-2.

In this scenario, in addition to the severe inflammatory picture, keep under control alterations in lipid and metabolic profiles could represent a good chance to predict severecritical outcomes and we agree with the pressing need to prospectively dissect the efficacy of statins in SARS-CoV-2.

Acknowledgements This study was partially supported by Regione Campania - Italy (POR Campania FESR 2014-2020 - ASSE I 2020, grant to Maurizio Bifulco and Patrizia Gazzerro). 


\section{Compliance with ethical standards}

Conflict of interest The authors declare that they have no conflict of interest.

Publisher's note Springer Nature remains neutral with regard to jurisdictional claims in published maps and institutional affiliations.

\section{References}

1. Guan, W.J., Ni, Z.Y., Hu, Y., Liang, W.H., Ou, C.Q., He, J.X., Liu, L., Shan, H., Lei, C.L., Hui, D.S.C., Du, B., Li, L.J., Zeng, G., Yuen, K.Y., Chen, R.C., Tang, C.L., Wang, T., Chen, P.Y., Xiang, J., Li, S.Y., Wang, J.L., Liang, Z.J., Peng, Y.X., Wei, L., Liu, Y., Hu, Y.H., Peng, P., Wang, J.M., Liu, J.Y., Chen, Z., Li, G., Zheng, Z.J., Qiu, S.Q., Luo, J., Ye, C.J., Zhu, S.Y., Zhong, N.S. Clinical characteristics of coronavirus disease 2019 in China. N. Engl. J. Med. (2020). https://doi.org/10.1056/NEJMoa2002032

2. M. Bifulco, P. Gazzerro, Statins in coronavirus outbreak: it's time for experimental and clinical studies. Pharmacol. Res. 156, 104803 (2020). https://doi.org/10.1016/j.phrs.2020.104803

3. Bifulco, M., Gazzerro, P. Statin therapy in COVID-19 infection: much more than a single pathway. Eur. Heart J. Cardiovas. Pharmacoth. (2020). https://doi.org/10.1093/ehjcvp/pvaa055

4. X.J. Zhang, J.J. Qin, X. Cheng, L. Shen, Y.C. Zhao, Y. Yuan, F. Lei, M.M. Chen, H. Yang, L. Bai, X. Song, L. Lin, M. Xia, F. Zhou, J. Zhou, Z.G. She, L. Zhu, X. Ma, Q. Xu, P. Ye, G. Chen, L. Liu, W. Mao, Y. Yan, B. Xiao, Z. Lu, G. Peng, M. Liu, J. Yang, L. Yang, C. Zhang, H. Lu, X. Xia, D. Wang, X. Liao, X. Wei, B.H. Zhang, X. Zhang, J. Yang, G.N. Zhao, P. Zhang, P.P. Liu, R. Loomba, Y.X. Ji, J. Xia, Y. Wang, J. Cai, J. Guo, H. Li, In-hospital use of statins is associated with a reduced risk of mortality among individuals with COVID-19. Cell Metab. 32(2), 176-187.e174 (2020). https://doi.org/10.1016/j.cmet.2020.06.015

5. A. De Spiegeleer, A. Bronselaer, J.T. Teo, G. Byttebier, G. De Tré, L. Belmans, R. Dobson, E. Wynendaele, C. Van De Wiele, F. Vandaele, D. Van Dijck, D. Bean, D. Fedson, B. De Spiegeleer, The effects of ARBs, ACEis, and statins on clinical outcomes of COVID-19 infection among nursing home residents. J. Am. Med. Dir. Assoc. 21(7), 909-914.e902 (2020). https://doi.org/10.1016/j. jamda.2020.06.018

6. X. Wei, W. Zeng, J. Su, H. Wan, X. Yu, X. Cao, W. Tan, H. Wang, Hypolipidemia is associated with the severity of COVID-19. J. Clin. Lipidol. 14(3), 297-304 (2020). https://doi.org/10.1016/j.jacl. 2020.04.008

7. B. Shen, X. Yi, Y. Sun, X. Bi, J. Du, C. Zhang, S. Quan, F. Zhang, R. Sun, L. Qian, W. Ge, W. Liu, S. Liang, H. Chen, Y. Zhang, J. Li, J. Xu, Z. He, B. Chen, J. Wang, H. Yan, Y. Zheng, D. Wang, J. Zhu, Z. Kong, Z. Kang, X. Liang, X. Ding, G. Ruan, N. Xiang, X. Cai, H. Gao, L. Li, S. Li, Q. Xiao, T. Lu, Y. Zhu, H. Liu, H. Chen, T. Guo, Proteomic and metabolomic characterization of COVID-19 patient sera. Cell 182(1), 59-72.e15 (2020). https://doi.org/10. 1016/j.cell.2020.05.032

8. B.J. Van Lenten, A.C. Wagner, G.M. Anantharamaiah, D.W. Garber, M.C. Fishbein, L. Adhikary, D.P. Nayak, S. Hama, M. Navab, A.M. Fogelman, Influenza infection promotes macrophage traffic into arteries of mice that is prevented by $\mathrm{D}-4 \mathrm{~F}$, an apolipoprotein A-I mimetic peptide. Circulation 106(9), 1127-1132 (2002). https://doi.org/10.1161/01.cir.0000030182.35880.3e

9. A.B. Chai, A.J. Ammit, I.C. Gelissen, Examining the role of ABC lipid transporters in pulmonary lipid homeostasis and inflammation. Respiratory Res. 18(1), 41 (2017). https://doi.org/10.1186/s12931017-0526-9 